\title{
Mood Disorder as the Presenting Manifestation of Demyelination
}

\author{
D. Tapos L. Sivaswamy \\ Department of Neurology, Children's Hospital of Michigan, Wayne State University School \\ of Medicine, Detroit, Mich., USA
}

\section{Key Words}

Multiple sclerosis $\cdot$ Adolescence $\cdot$ Mood disorder

\begin{abstract}
Objective: To report an unusual presenting manifestation of multiple sclerosis in a teenager. Case Report: We report the case of a 16-year-old male who was diagnosed with schizoaffective disorder a year prior to his neurological consultation. Neurological examination showed evidence of mild lower extremity spasticity with ataxia and dysarthria. Imaging revealed lesions suggestive of multiple sclerosis. Conclusion: An organic basis for psychiatric disorders is often overlooked. Children presenting with symptoms suggestive of a mood disorder, decline in cognition or psychosis deserve a detailed neurological examination and appropriate imaging if indicated, before starting them on antidepressant or neuroleptic medication.
\end{abstract}

\section{Introduction}

Most young adults with psychosis have a nonorganic etiology for their symptoms, with schizophrenia and bipolar affective disorders accounting for the majority of cases. However, in $5-10 \%$ of cases there is an organic basis that might include stroke, inborn errors of metabolism or demyelinating lesions. Recognition of these conditions is often possible by eliciting relevant historical facts and pertinent neurological signs. It is vital to be able to recognize these entities early in the disease process to initiate appropriate treatment options and offer counseling regarding prognosis. We present the case of a teenager who was diagnosed as having a schizoaffective disorder but noted to have unusual physical findings. This led to imaging studies and a final diagnosis of multiple sclerosis (MS). 
Tapos et al.: Mood Disorder as the Presenting Manifestation of Demyelination

\section{Case Report}

A 16-year-old Caucasian adolescent with a history of emotional lability for a period of 12 months presented to the neurology clinic for an evaluation. He had bouts of intense sadness characterized by withdrawal from routine activities, inability to attend school and suicidal thoughts. These episodes were interspersed with periods of aggressive outbursts, depersonalization and incoherent thinking during which time he experienced auditory hallucinations. The patient also did not wish to participate in soccer - a game he had previously been passionate about. He was evaluated by a psychiatrist about 6 months after the onset of his symptoms. He was diagnosed as having a schizoaffective disorder and started on antipsychotic medication. Counseling services were also commenced soon after seeing the psychiatrist. Subsequently, marginal improvement in behavior was reported by the family. The patient was referred to the child neurology clinic by his pediatrician for a neurological 'checkup'.

On detailed questioning he admitted to an 'attack' of clumsiness a few months prior to presentation while playing soccer. He felt unsteady on his legs for a period of 2 days and never felt comfortable returning to the game. He also noted that walking up and down stairs was strenuous now with myalgia in his calves. He mentioned that he had to take special education classes for the past few months and in fact was attending summer school to make up for a recent decline in grades. There was no history of loss of vision, diplopia, dysphagia, bladder/bowel disturbances, fatigue or sensory symptoms.

Examination revealed a well-built young man in no apparent distress. He appeared well groomed. He was not enthusiastic to answer questions pertaining to his health and replied in monosyllables while avoiding eye contact. His speech was noted to be dysarthric. Of note, there was mild spasticity of his lower extremities demonstrable only by stiffness at both ankles. He had exaggerated deep tendon reflexes at his knees and ankles. His gait was wide based and unsteady and he had difficulty with tandem gait. He could not hop on one foot, and running was clumsy. Plantar response was upgoing on both sides. He had difficulty reading from a grade-level book and displayed poor comprehension of the content. Slow information processing and poor memory retrieval were present as well. Results of the Wechsler Intelligence Scale for Children - Fourth Edition (WISC-IV) and Gordon Diagnostic System for executive functioning are presented in table 1 and table 2, respectively. On a selfreport behavioral measure, utilizing the Youth Self Report questionnaire, the patient showed a number of internalizing problems (anxiety, depression and thought problems).

In view of the constellation of symptoms suggestive of a disorder involving cognition and mood, and physical findings indicative of an upper motor neuron disease process, magnetic resonance imaging (MRI) of the brain was ordered. Imaging revealed several T2fluid-attenuated inversion recovery (FLAIR) hyperintense signals in the white matter adjacent to the ventricles (fig. 1a). Lesions were present in the cerebellum as well (fig. 1b). Following administration of contrast material, some of the areas showed enhancement suggestive of an active demyelinating disease process. Subsequently, MRI of the spine was obtained, which also showed lesions suggestive of demyelination in the thoracic spine (fig. 2).

A comprehensive workup was initiated starting with spinal fluid analysis which yielded a white blood cell count of $1 \mathrm{cell} / \mathrm{mm}^{3}$, red blood cell count of 9 cells $/ \mathrm{mm}^{3}$, protein level was $69 \mathrm{mg} / \mathrm{dl}$, glucose level was $54 \mathrm{mg} / \mathrm{dl}$, 9 oligoclonal bands were detected compared to 0 oligoclonal bands in the serum, and IgG index was 0.93 with a normative value of less than 0.85. Testing for bacteria in blood and spinal fluid did not yield positive results. Viral titers for adenovirus, enterovirus, equine encephalitides and borreliosis were unremarkable. 
Tapos et al.: Mood Disorder as the Presenting Manifestation of Demyelination

Serum testing for antinuclear antibodies, antibodies against double-stranded DNA, lupus anticoagulant, angiotensin-converting enzyme, vitamin $\mathrm{B}_{12}$ levels and thyroid antibodies were negative. Human immunodeficiency virus testing was negative, as were tests for syphilis and neurosarcoidosis.

A diagnosis of MS was made by applying the McDonald criteria that utilize findings on MRI and clinical symptomatology [1]. The patient was started on glatiramer acetate at a dose of $20 \mathrm{mg}$ per day. He tolerated it well and has not had any clinical relapses, psychiatric or otherwise, since therapy was commenced. He has also been weaned off his psychotropic medications with no further exacerbation of his schizoaffective disorder. The patient continues to receive special education classes through school.

\section{Discussion}

The prevalence of pediatric MS is $0.9 / 100,000$. Onset in childhood occurs in $2.7-10 \%$ of all diagnosed cases, with most patients being female [2,3]. MS most commonly affects adults in the 2nd-4th decades of life. A higher proportion of African-American and Hispanic patients have childhood onset of their disease. Optic neuritis is the most common presenting manifestation of the disease in childhood [4]. Similar to MS in adults, common clinical features at the time of initial presentation in childhood include motor weakness, sensory disturbances and symptoms suggestive of cerebellar disease (ataxia, dysarthria). Psychiatric symptoms as the initial clinical feature are a rare phenomenon and are believed to occur in less than $2 \%$ of patients [4]. Nonetheless, it has been noted in one study of patients diagnosed with MS that $25 \%$ had a depressive period prior to the diagnosis, as opposed to $17 \%$ in a control group. Hence, it may be that disorders of mood and personality may be fairly frequent even at the time of initial diagnosis, though they may not trigger a comprehensive neurological workup [5].

The psychiatric manifestations of pediatric MS are poorly reported. Extrapolation of adult MS literature suggests that neuropsychiatric symptoms of MS can be grouped into 2 categories: cognitive and mood. Amongst the latter, the most widely noted are depression, bipolar disease and pseudobulbar affect. Other psychiatric symptoms include irritability, apathy, disinhibition and euphoria [6]. Individuals with MS have a $50 \%$ lifetime prevalence of depression, with suicide rates that are higher compared to the general population. Bipolar disease is also 2-3 times more common in this population [7, 8]. Pseudobulbar affect is more common in those with chronic MS with significant cognitive impairment and hence is unlikely to occur in childhood.

The etiology of the psychiatric features is not completely elucidated. There is evidence to suggest that patients with high lesion load in the temporal lobes, especially the left temporal lobe, are more likely to have psychiatric features [9]. Inflammatory cytokines, which mediate disease pathology in MS, especially interferon-alpha and interleukin-1, are known to have an effect on the serotonin and noradrenergic systems of the central nervous system and can mediate behavior and mood. Furthermore, decrease of inflammatory cells and cytokines by use of immune-modulating agents has been shown to positively affect psychiatric symptomatology [6]. Other potential etiologies can include adverse effects of medication such as steroids and immune modulators, or it may be that MS and mood disorders share a common genetic link.

Cognitive deficits occur in at least half of all adult individuals with MS. Recent studies have highlighted deficits in attention, executive function and working memory in the pediatric age group, even in the absence of significant physical impairment. Such cognitive 
loss may be a consequence of disease affecting the maturation process of the white matter pathways in the frontal lobe [10]. Children diagnosed with MS require objective tests of cognition at regular intervals, as cognitive dysfunction may exhibit a linear decline, in parallel with their neurological impairment.

A diagnosis of MS can be made on clinical parameters alone. However, MRI techniques have revolutionized the clinicians' approach to this disorder in the pediatric age group, adding a high degree of specificity and sensitivity and allowing for earlier diagnosis. The McDonald criteria and KIDMUS MRI criteria are 2 such well-validated measures to arrive at a conclusive diagnosis [11].

An organic basis for psychiatric disorders in childhood is often overlooked for extended periods of time, as a detailed neurological examination may not be performed by the clinician. Adults with MS whose initial clinical symptoms were purely psychiatric in nature have been reported [12]. To our knowledge this is the first case to be reported in the literature on an individual less than 18 years of age.

In conclusion, children presenting with disorders of mood and affect deserve a thorough neurological examination before commencing them on psychotropic medications. Performing routine imaging in the form of MRI or computerized tomography in patients with psychosis and mood variability, but with no pertinent historical findings and nonlocalizing examination, is unlikely to be helpful [13]. Treatment options include use of atypical antipsychotics such as olanzapine or ziprasidone, in addition to immune-modulating therapy directed towards the underlying disease process [14]. Last but not least, other diseases may present with psychiatric disturbances in early adulthood including inborn errors of metabolism such as urea cycle disorders, porphyria, adrenoleukodystrophy, Niemann-Pick type C, Wilson disease [15], low grade gliomas of the left temporal lobe and localizationrelated epilepsy.

\section{References}

1 Polman CH, Reingold SC, Banwell B, Clanet M, Cohen JA, Filippi M, Fujihara K, Havrdova E, Hutchinson M, Kappos L, Lublin FD, Montalban X, O'Connor P, Sandberg-Wollheim M, Thompson AJ, Waubant E, Weinshenker B, Wolinsky JS: Diagnostic criteria for multiple sclerosis: 2010 revisions to the McDonald criteria. Ann Neurol 2011;69:292-302.

-2 Duquette P, Murray TJ, Pleines J, Ebers GC, Sadovnick D, Weldon P, Warren S, Paty DW, Upton A, Hader W, et al: Multiple sclerosis in childhood: clinical profile in 125 patients. J Pediatr 1987;111:359-363.

- 3 Simone IL, Carrara D, Tortorella C, Liguori M, Lepore V, Pellegrini F, Bellacosa A, Ceccarelli A, Pavone I, Livrea P: Course and prognosis in early-onset MS: comparison with adult onset forms. Neurology 2002;59:1922-1928.

4 Chitnis T, Glanz B, Jaffin S, Healy B: Demographics of pediatric-onset multiple sclerosis in an MS center population from the Northeastern United States. Mult Scler 2009;15:627-631.

5 Lo Fermo S, Barone R, Patti F, Laisa P, Cavallaro TL, Nicoletti A, Zappia M: Outcome of psychiatric symptoms presenting at onset of multiple sclerosis: a retrospective study. Mult Scler 2010;16:742-748.

-6 Sullivan J, Weinshenker B, Mikail S, et al: Depression before and after diagnosis of multiple sclerosis. Mult Scler 1995;1:104-108.

7 Haussleiter IS, Brüne M, Juckel G: Psychopathology in multiple sclerosis: diagnosis, prevalence and treatment. Ther Adv Neurol Disord 2009;2:13-29.

8 Patten SB, Beck CA, Williams JV, Barbui C, Metz LM: Major depression in multiple sclerosis. A populationbased perspective. Neurology 2003;61:1524-1527.

9 Marrie RS, Horwitz R, Cutter G, et al: The burden of medical comorbidity in multiple sclerosis: frequent, underdiagnosed and undertreated. Mult Scler 2009;15:385-392.

10 MacAllister WS, Christodoulou C, Milazzo M, Krupp LB: Longitudinal neuropsychological assessment in pediatric multiple sclerosis. Dev Neuropsychol 2007;32:625-644.

11 Kurne A, Oguz KK, Oz Aksu A, Yarar C, Duman 0, Kurul SH, Serdaroglu A, Anlar B: Magnetic resonance imaging at first episode in pediatric multiple sclerosis retrospective evaluation according to KIDMUS and lesion dissemination in space criteria. Brain Dev 2010;32:487-494. 
12 Fazzito MM, Jordy SS, Tilbery CP: Psychiatric disorders in multiple sclerosis patients. Arq Neuropsiquiatr 2009;67:664-667.

13 Albon E, Tsourapas A, Frew E, Davenport C, Oyebode F, Bayliss S, Arvanitis T, Meads C: Structural neuroimaging in psychosis: a systematic review and economic evaluation. Health Technol Assess 2008;12:1-184.

14 Habek M, Brinar M, Brinar VV, Poser C: Psychiatric manifestations of multiple sclerosis and acute disseminated encephalomyelitis. Clin Neurol Neurosurg 2006;108:290-294.

15 Sedel F, Baumann N, Turpin JC, Lyon-Caen O, Saudubray JM, Cohen D: Psychiatric manifestations revealing inborn errors of metabolism in adolescents and adults. J Inherit Metab Dis 2007;30:631-641.

Table 1. WISC-IV indicating factor score results within low average range

\begin{tabular}{lll}
\hline & Standard score & Description \\
\hline Verbal comprehension index & 81 & Low average \\
Perceptual reasoning index & 85 & Low average \\
Working memory index & 80 & Low average \\
Processing speed index & 82 & Low average \\
Full scale IQ & 80 & Low average \\
\hline
\end{tabular}

Table 2. Gordon Diagnostic System for executive functioning

\begin{tabular}{lll}
\hline & T score & Description \\
\hline Sustained attention (hits) & 49 & Average \\
Impulse control (false alarm) & 40 & Low average \\
\hline
\end{tabular}




\section{Case Reports in Neurology}

\begin{tabular}{l|l}
\hline Case Rep Neurol 2013;5:104-109 & \\
\hline DOI: 10.1159/000351282 & $\begin{array}{l}\text { @ 2013 S. Karger AG, Basel } \\
\text { www.karger.com/crn }\end{array}$ \\
\hline
\end{tabular}

Tapos et al.: Mood Disorder as the Presenting Manifestation of Demyelination
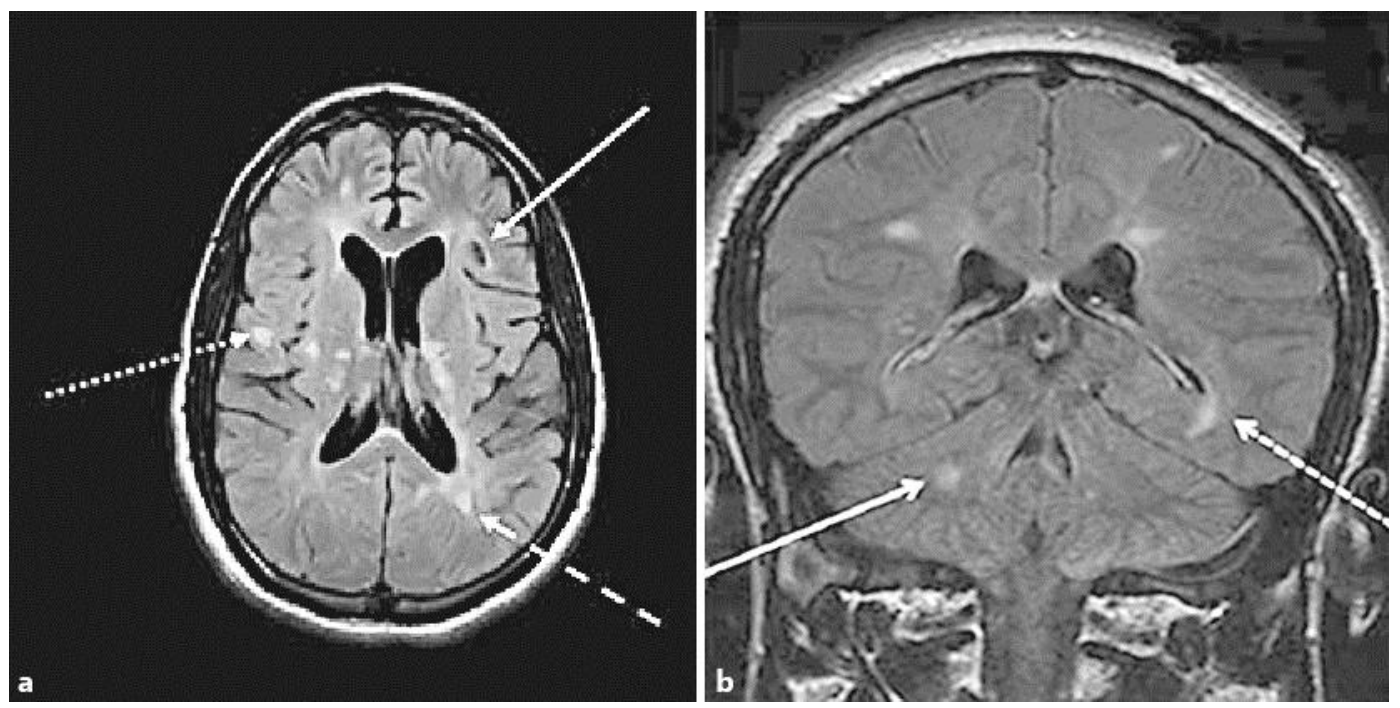

Fig. 1. a Axial FLAIR MRI sequence demonstrating hyperintense lesions in the right temporal region (dotted arrow), a lesion in the left periventricular white matter (dashed arrow) and a 'dark hole' (solid arrow) in the left frontal region. The lesions in the frontal and temporal regions might account for the patient's psychiatric manifestations. b Coronal FLAIR MRI sequence with bright demyelinating plaques in the right cerebellum (solid arrow) and left periventricular area (dashed arrow). The lesion in the cerebellum might account for the patient's dysarthria and poor coordination.

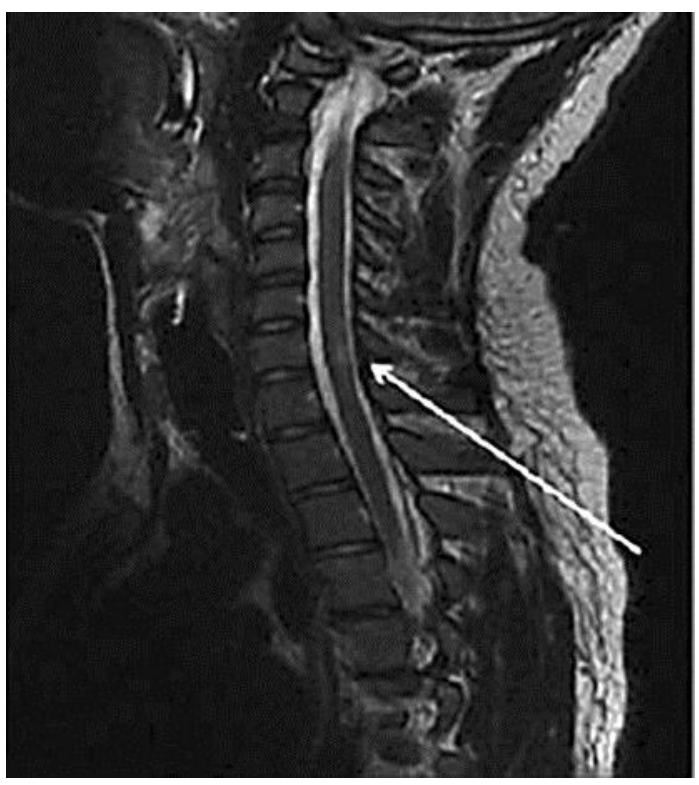

Fig. 2. Sagittal FLAIR sequence of the cervical and thoracic spine with a demyelinating lesion in the upper thoracic area (arrow). 